\title{
Non-proper helicoid-like limits of closed minimal surfaces in 3-manifolds
}

\author{
Maria Calle • Darren Lee
}

Received: 6 February 2008 / Accepted: 29 February 2008 / Published online: 4 April 2008

(C) The Author(s) 2008

\begin{abstract}
We show that there exists a metric with positive scalar curvature on $\mathbf{S}^{2} \times \mathbf{S}^{1}$ and a sequence of embedded minimal cylinders that converges to a minimal lamination that, in a neighborhood of a strictly stable 2 -sphere, is smooth except at two helicoid-like singularities on the 2-sphere. The construction is inspired by a recent example by D. Hoffman and B. White.
\end{abstract}

\section{Introduction}

Roughly speaking, it is expected that the only two types of singular laminations that can occur as limits of sequences of closed embedded minimal surfaces in a 3-manifold with positive scalar curvature are accumulations of catenoids and non-proper helicoid-like limits. Recall that a lamination is a foliation that does not necessarily fill the entire space; in particular, just like in a foliation, the leaves of a lamination must be locally parallel graphs. An example of the first type of limit was constructed by Colding and De Lellis in [5]. Prior to the construction given here, no non-proper helicoid-like limits were known to exist as limits of closed surfaces. We construct such a limit in $\mathbf{S}^{2} \times \mathbf{S}^{1}$ where two helicoid-like singularities lie on a strictly stable 2-sphere.

For closed Riemannian manifolds with positive Ricci curvature, combining the work of Choi and Wang [4] and Yang and Yau [45] gives an area bound for embedded minimal surfaces that depends only on the lower bound for the Ricci curvature of the manifold and on the genus of the surface. In particular, constructions such as those in [5] and in this paper are

\footnotetext{
M. Calle ( $\varangle)$

Max Planck Institute for Gravitational Physics, Albert Einstein Institute,

Am Mühlenberg 1, 14476 Golm, Germany

e-mail: maria.calle@aei.mpg.de

D. Lee

Courant Institute of Mathematical Sciences, 251 Mercer Street, New York, NY 10012, USA

e-mail: leed@cims.nyu.edu
} 


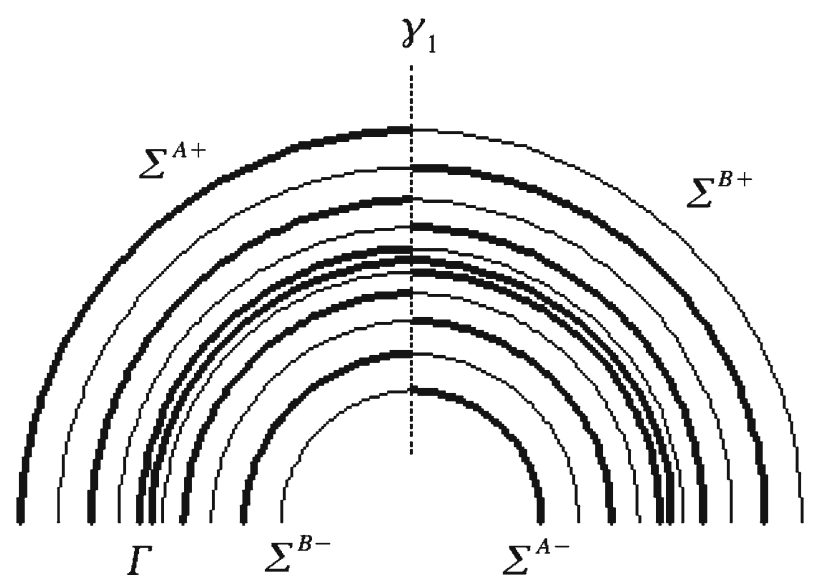

Fig. 1 A schematic cross-section near one of the helicoid axes of the limit lamination in Theorem 1. The limit consists of the strictly stable 2 -sphere $\Gamma$ and four embedded minimal disks joined along the central axis $\gamma_{1} . \Sigma^{A+}$ and $\Sigma^{B+}$ lie on one side of $\Gamma$ and $\Sigma^{A-}$ and $\Sigma^{B-}$ lie on the other side. Each of the four spirals infinitely into $\Gamma$. These surfaces connect to a symmetric configuration around a second axis $\gamma_{2}$

not possible in manifolds of positive Ricci curvature as both of these constructions require sequences of closed minimal surfaces with unbounded area. See also [3], where Choi and Schoen proved that any limit of closed minimal surfaces in a closed 3-manifold with positive Ricci curvature must be smooth.

An immersed surface $\Sigma \subset M$ is said to be proper if the intersection of $\Sigma$ with any compact subset of $M$ is compact. Similarly, a lamination is proper if each leaf is proper. The study of the properness of minimal surfaces in Euclidean 3-space has a long history; see in particular the work on the Calabi-Yau conjectures [1,12,18,30,31,39]. For limit laminations in Euclidean 3-space, many results about properness are known. In particular, Colding and Minicozzi showed in [13-16] that any sequence of embedded minimal disks in balls of increasing, unbounded radius in $\mathbf{R}^{3}$ must converge off of a curve to a foliation of planes, and so non-proper limits are impossible. However, in [8], Colding and Minicozzi construct a sequence of embedded minimal disks in a fixed ball in $\mathbf{R}^{3}$ with boundaries in the boundary of the ball that converges to a limit which is not proper; see also [32,33].

Our construction is inspired by a beautiful variational construction by Hoffman and White [28] of the genus one helicoid; see also the earlier known constructions of the genus one helicoid $[26,27]$.

We consider the case of a manifold which is topologically $\mathbf{S}^{2} \times \mathbf{S}^{1}$, for which we have the following theorem:

Theorem 1 There exists a metric with positive scalar curvature on $M=\mathbf{S}^{2} \times \mathbf{S}^{1}$ and a sequence of embedded minimal cylinders $\left\{\Sigma_{n}\right\}$ with boundary in an unstable 2-sphere that, in a neighborhood of a strictly stable 2 -sphere $\Gamma$, converges to a minimal lamination that is smooth except at two helicoid-like singularities on $\Gamma$.

Specifically, in a neighborhood $\Omega$ of the strictly stable 2-sphere, $\Sigma_{n} \cap \Omega \backslash \Gamma$ converges to two non-properly embedded minimal surfaces $\Sigma^{ \pm}$, one on each side of $\Gamma$, with $\overline{\Sigma^{ \pm}} \backslash \Sigma^{ \pm}=\Gamma$. Furthermore, away from two axes, $\Sigma^{ \pm}$is the union of two embedded minimal disks $\Sigma^{\{A, B\} \pm}$, each of which spirals into $\Gamma$ (see Fig. 1). 

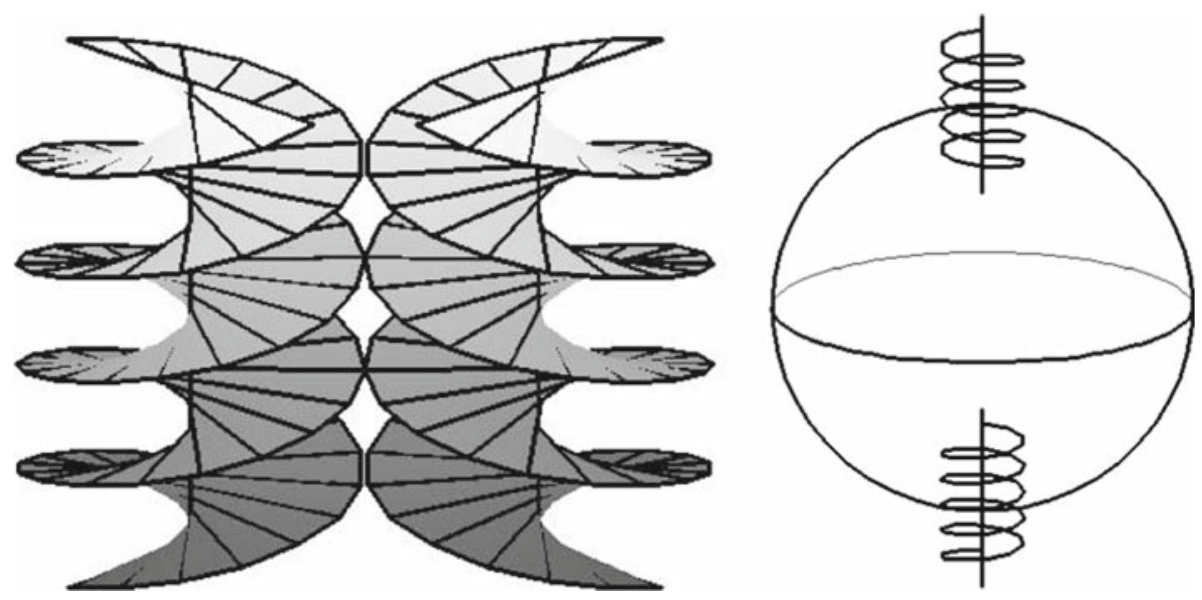

Fig. 2 On the left, two helicoids with opposite orientation glued together. On the right, two helicoids spiral around the polar axes of $\mathbf{S}^{2}$

The metric in Theorem 1 will be a warped product metric with particular assumptions about the warping factor. If we instead use the product metric on $\mathbf{S}^{2} \times \mathbf{S}^{1}$ (where each $\mathbf{S}^{2} \times\{z\}$ is minimal but not strictly stable), the same construction will give us a sequence of embedded minimal cylinders that converges smoothly away from two circles to the smooth foliation of $\mathbf{S}^{2} \times \mathbf{S}^{1}$ by the parallel minimal spheres $\mathbf{S}^{2} \times\{z\}$. Each surface in this sequence looks like it is obtained by gluing together two oppositely oriented helicoids. Each helicoid has a circular axis $\{x\} \times \mathbf{S}^{1}$ and $\{-x\} \times \mathbf{S}^{1}$, which are orthogonal to each 2-sphere $\mathbf{S}^{2} \times\{z\}$ and are "antipodal" (they go through $x$ and $-x$, respectively).

In Sect. 3 we will construct the sequence of minimal surfaces $\Sigma_{n}$. The surfaces in our sequence will be embedded minimal cylinders that spiral an increasing number of times around two polar axes $\gamma_{1}$ and $\gamma_{2}$ of the form $\{x\} \times \mathbf{S}^{1}$. They spiral around the axes in opposite directions with the corresponding layers connected (see Fig. 2). In order to find these surfaces, we wish to solve Plateau problems in the universal cover $\Omega^{\prime}$ of $\Omega \backslash\left(\gamma_{1} \cup \gamma_{2}\right)$, where $\Omega$ is a neighborhood of a strictly stable 2 -sphere $\Gamma$.

After constructing the sequence, in Sect. 4 we will take the limit when $n \rightarrow \infty$ and obtain a minimal lamination. By choosing a sufficiently small neighborhood of the strictly stable 2 -sphere, we can assume that $\Gamma$ is the only closed minimal surface in $\Omega$. Thus, this lamination must consist of the strictly stable 2-sphere and two minimal surfaces, one on each side, that spiral into it. In particular, these two minimal surfaces will be embedded but not proper.

\section{Preliminaries}

We parametrize $M=\mathbf{S}^{2} \times \mathbf{S}^{1}$ by $(\phi, \theta, z) \in[0, \pi] \times[0,2 \pi) \times(-\pi, \pi]$, where $(\phi, \theta)$ are latitudinal and longitudinal coordinates on $\mathbf{S}^{2}$ and $z \in(-\pi, \pi] \sim \mathbf{S}^{1}$. We consider a warped product metric in $M$ given by

$$
g^{2}=\omega^{2}(z)\left(d \phi^{2}+\sin ^{2} \phi d \theta^{2}\right)+d z^{2} .
$$

We will assume that $\omega$ is a smooth function $\mathbf{S}^{1} \rightarrow \mathbf{R}$ such that:

(i) $\omega(\pi)>\omega(0)=1$, 
(ii) $\omega(z)=\omega(-z)$,

(iii) $\quad \omega^{\prime \prime}(0)>0$, and

(iv) $z=0, \pi$ are the only critical points of $\omega$.

With these assumptions, $\Gamma=\{z=0\}$ is a strictly stable minimal 2-sphere and $\{z=\pi\}$ is an unstable minimal 2-sphere. We will be working in the neighborhood $\Omega=M \backslash\{z=\pi\}$ of the strictly stable 2 -sphere $\Gamma$. Note that the boundary $\partial \Omega=\mathbf{S}^{2} \times\{\pi,-\pi\}$ is minimal and hence weakly mean convex. Recall that $\partial \Omega$ is weakly mean convex if its mean curvature is $H \geq 0$ with respect to the unit normal vector pointing towards $\Omega$. Also, $\Gamma$ is the only closed minimal surface in $\Omega$ :

Lemma 2 If $\Sigma$ is a closed minimal surface in $M$ away from $\{z=\pi\}$, then $\Sigma=\Gamma$.

In order to prove this lemma, we will need the following maximum principle of Solomon and White (see [42]):

Theorem 3 Let $\Omega$ be open and $\Sigma_{1}$ and $\Sigma_{2}$ be connected minimal surfaces in $\Omega$, and suppose that $\Sigma_{1}$ is smooth. Further suppose that $\Sigma_{1}$ lies weakly on one side of $\Sigma_{2}$ (that is, $\Sigma_{1}$ is contained in the closure of one of the components of $\left.\Omega \backslash \Sigma_{2}\right)$. Then the two surfaces either coincide (i.e., are either the same surface or are each subsets of a single larger minimal surface) or are disjoint.

The conclusion also holds when $\Sigma_{2}$ is mean-convex, that is, its mean curvature vector points (where it is not 0 ) into the component of $\Omega \backslash \Sigma_{2}$ containing $\Sigma_{1}$.

Proof of Lemma 2 First, we observe that each surface $\Gamma_{a}=\{z=a\}$ with $a \neq 0, \pi$ is strictly mean convex, with mean curvature vector pointing towards the strictly stable 2 -sphere $\Gamma$. If $0<a<\pi$, consider the unit normal vector $\vec{n}_{\Gamma_{a}}=-\frac{\partial}{\partial z}$ pointing towards $\Gamma$. We can extend this vector to a vector field $X=-\frac{\partial}{\partial z}$ defined on $M$, and then we can compute the mean curvature of $\Gamma_{a}$ with respect to $\vec{n}_{\Gamma_{a}}$ as:

$$
\begin{aligned}
H_{\Gamma_{a}} & =-\frac{1}{2} \operatorname{div}_{M} X \\
& =-\left.\frac{1}{2} \frac{\partial}{\partial z}\right|_{z=a}(-\ln (\operatorname{det} g)) \\
& =\left.\frac{1}{2} \frac{\partial}{\partial z}\right|_{z=a}\left(\ln \left(\omega^{4}(z) \sin ^{2}(\phi)\right)\right) \\
& =2 \frac{\omega^{\prime}(a)}{\omega(a)}>0 .
\end{aligned}
$$

If $-\pi<a<0$, the same argument applied to the unit normal vector $\vec{n}_{\Gamma_{a}}=\frac{\partial}{\partial z}$ shows that the surface is mean convex.

Suppose now that $\Sigma$ is a closed minimal surfaces away from $\{z=\pi\}$ and different from $\Gamma$. Then there is a value $0<a<\pi$ such that $\Sigma \subset\{-a \leq z \leq a\}$ and either $\Sigma \cap \Gamma_{a} \neq \emptyset$ or $\Sigma \cap \Gamma_{-a} \neq \emptyset$. But this contradicts the maximum principle (Theorem 3), as $\Gamma_{ \pm a}$ is strictly mean convex.

The surfaces in our sequence will be embedded minimal cylinders with boundary in $\{z=\pi\}$ that spiral an increasing number of times around the polar axes $\gamma_{1}=\{\phi=0\}$ and $\gamma_{2}=\{\phi=\pi\}$. In order to find these surfaces, we wish to solve Plateau problems in the universal cover $\Omega^{\prime}$ of $\Omega \backslash\left(\gamma_{1} \cup \gamma_{2}\right)$. In particular, we will use the following theorem of Meeks and Yau (Theorem 5 of [37]): 
Theorem 4 Let $M$ be a compact Riemannian 3-manifold, and suppose that $M$ can be isometrically embedded in a larger manifold $\tilde{M}$ such that $\partial M$ is a two-dimensional subcomplex of $\tilde{M}$ consisting of smooth two-dimensional simplexes $\left\{\Lambda_{1}, \ldots, \Lambda_{k}\right\}$ with the following properties:

(i) Each $\Lambda_{i}$ is a smooth surface in $\tilde{M}$ whose mean curvature is non-negative with respect to the outward normal.

(ii) Each surface $\Lambda_{i}$ is a compact subset of some smooth surface $\tilde{\Lambda}_{i} \subset \tilde{M}$ where $\tilde{\Lambda}_{i} \cap M=$ $\Lambda_{i}$ and $\partial \tilde{\Lambda}_{i} \subset \partial \tilde{M}$.

Let $N$ be a compact subdomain of $\partial M$ such that each homotopically nontrivial closed curve in $N$ is also homotopically nontrivial in $M$. Then there is a stable minimal embedding $f: N \rightarrow M$ so that $f(\partial N)=\partial N$.

The stable minimal surface constructed in this theorem is, in fact, area-minimizing, as shown in the proof in [37]. With the help of this theorem, we will construct a sequence of embedded minimal disks that are smooth in the interior. Then we will apply the following result of Hardt and Simon to prove regularity of the boundary (Theorem 11.1 in [23]):

Theorem 5 Let $U$ be an area-minimizing surface and $\partial U$ be a connected oriented embedded $C^{1, \alpha}$ curve. Then $U$ is a connected embedded $C^{1, \alpha}$ hypersurface with boundary.

This construction gives us half of the cylinders $\Sigma_{n}$. Then, we will use the Schwarz reflection principle (Lemma 7.3 in [40]) to reflect them across the axes $\gamma_{1}$ and $\gamma_{2}$ :

Theorem 6 Suppose $\Sigma$ is a minimal surface in a 3-manifold $M$ whose boundary contains a geodesic segment $\gamma$. Suppose additionally that there exists an isometry $G$ in $M$ whose fixed points include $\gamma$. Then $\Sigma$ can be extended to a minimal surface $\Sigma \cup G(\Sigma)$ symmetric across $\gamma$.

We recall that a codimension one lamination on a 3-manifold $M^{3}$ is a collection $\mathcal{L}$ of smooth disjoint surfaces (called leaves) such that $\bigcup_{\Lambda \in \mathcal{L}} \Lambda$ is closed, and such that for each $x \in M$ there exists an open neighborhood $U$ of $x$ and a coordinate chart $(U, \Phi)$ with $\Phi(U) \subset \mathbf{R}^{3}$ so that in these coordinates the leaves in $\mathcal{L}$ pass through $\Phi(U)$ in slices of the form $(\mathbf{R} \times\{t\}) \cap \Phi(U)$. A minimal lamination is a lamination whose leaves are minimal. Note that any (compact) embedded surface is a lamination. When proving the convergence of our sequence, we will need the following proposition (Proposition B.1 in [16]):

Proposition 7 Let $M^{3}$ be a fixed 3-manifold. If $\mathcal{L}_{i} \subset B_{2 R}(x) \subset M$ is a sequence of minimal laminations with uniformly bounded curvatures (where each leaf has boundary contained in $\partial B_{2 R}(x)$ ), then a subsequence $\mathcal{L}_{j}$ converges in the $C^{\alpha}$ topology for any $\alpha<1$ to a (Lipschitz) lamination $\mathcal{L}$ in $B_{R}(x)$ with minimal leaves.

\section{The sequence of embedded minimal surfaces $\left\{\Sigma_{n}\right\}$}

We consider $\Omega^{\prime}$ the universal cover of $\Omega \backslash\left(\gamma_{1} \cup \gamma_{2}\right)$. We can parametrize this universal cover as $\Omega^{\prime}=[0, \pi] \times \mathbf{R} \times(-\pi, \pi)$. In these coordinates, the metric in $\Omega^{\prime}$ is given by the same expression as the original metric in $M$. The topological boundary of $\Omega^{\prime}$ is $\partial \Omega^{\prime}=\{\phi=$ $0\} \cup\{\phi=\pi\} \cup\{z=\pi\} \cup\{z=-\pi\}$, and the two pieces $\{z=\pi\}$ and $\{z=-\pi\}$ are mean-convex.

The main difficulty in applying Theorem 4 is the portion of the boundary corresponding to $\gamma_{1}$ and $\gamma_{2}$, as the warped product metric in $\Omega^{\prime}$ becomes degenerate as we approach these axes. 
In order to apply Meeks-Yau, we will remove small $\epsilon$-tubes around $\gamma_{1}$ and $\gamma_{2}$, then smoothly glue together these new ends. The resulting manifold will be topologically a solid torus and will only have mean-convex boundary. Thus, we can solve Plateau problems in a sequence of these manifolds with $\epsilon \rightarrow 0$. Subsets of the area-minimizing solutions in this sequence can be isometrically embedded in $\Omega^{\prime}$, and projecting back to $\Omega$ we can get a subsequence converging to an embedded solution to the original Plateau problem in $\Omega^{\prime}$.

The precise construction is as follows.

For each $\epsilon>0$, let $\Omega_{\epsilon}^{\prime}$ be the universal cover of $\Omega$ minus the $\epsilon$-tubes around $\gamma_{1}$ and $\gamma_{2}$. Thus,

$$
\Omega_{\epsilon}^{\prime}=(\epsilon, \pi-\epsilon) \times \mathbf{R} \times(-\pi, \pi) \subset \Omega^{\prime} .
$$

We can isometrically embed $\Omega_{\epsilon}^{\prime}$ into the manifold $N_{\epsilon}=\mathbf{S}^{1} \times \mathbf{R} \times[-\pi, \pi]$ endowed with the metric

$$
g_{\epsilon}^{2}=\omega^{2}(z)\left(d \phi^{2}+\alpha_{\epsilon}^{2}(\phi) d \theta^{2}\right)+d z^{2}
$$

where $\alpha_{\epsilon}: \mathbf{S}^{1}=[0, \pi) \rightarrow \mathbf{R}$ is a smooth function satisfying:

(i) $\alpha_{\epsilon}(\phi)=\sin (\phi)$ for $\phi \in[\epsilon, \pi-\epsilon]$.

(ii) $\alpha_{\epsilon}^{\prime}(\phi)>0$ for $0<\phi<\frac{\pi}{2}$ and $\alpha_{\epsilon}^{\prime}(\phi)<0$ for $\frac{\pi}{2}<\phi<\pi$.

(iii) $\alpha_{\epsilon}(\phi)>0$ for all $\phi \in \mathbf{S}^{1}$.

Conceptually, we imagine $\alpha_{\epsilon}(\phi)$ as a smooth version of $f(\phi)=\max \{\sin (\phi), \sin (\epsilon)\}$. This metric glues together the $\epsilon$-tubes around $\gamma_{1}$ and $\gamma_{2}$.

We next construct embedded minimal surfaces in $N_{n, \epsilon}=\mathbf{S}^{1} \times[-n \pi, n \pi] \times[-\pi, \pi] \subset N_{\epsilon}$ with boundary $\gamma_{3}=\{(\phi,-n \pi,-\pi)\}$ and $\gamma_{4}=\{(\phi, n \pi, \pi)\}$ using Theorem 4 . To do this, we first need to show that the boundary of $N_{n, \epsilon}$ is mean convex:

Lemma 8 The boundary $\partial N_{n, \epsilon}$ satisfies the hypothesis of Theorem 4. In particular, it is contained in the union of a finite number of minimal surfaces that meet at an angle of $\frac{\pi}{2}$.

Proof First, observe that the boundary of $N_{n, \epsilon}$ consists of the "horizontal" surfaces $\Lambda_{1}=$ $\{z=-\pi\}$ and $\Lambda_{2}=\{z=\pi\}$ and the "vertical" surfaces $\Lambda_{3}=\{\theta=-n \pi\}$ and $\Lambda_{4}=\{\theta=$ $n \pi\}$, and neither the two vertical pieces nor the two horizontal pieces intersect. Therefore, to satisfy the intersection requirement, we only need to look at the intersection of a horizontal surface and a vertical surface.

Let $x \in \Lambda_{1} \cap \Lambda_{3}$. Then $\frac{\partial}{\partial \phi} \in T_{x}\left(\Lambda_{1} \cap \Lambda_{3}\right)$, so the angle between the surfaces is the angle between $\frac{\partial}{\partial \theta} \in T_{x}\left(\Lambda_{1}\right)$ and $\frac{\partial}{\partial z} \in T_{x}\left(\Lambda_{3}\right)$. Direct computation shows that these two vectors are orthogonal, and so the surfaces meet orthogonally. The same argument shows that the other three pairs of surfaces also intersect orthogonally. In particular, this shows that if we embed $N_{n, \epsilon}$ in a larger manifold and extend each $\Lambda_{i}$, the extension will lie outside of $N_{n, \epsilon}$, as required in (ii) in Theorem 4.

We will next show that the horizontal surfaces $\Lambda_{1}$ and $\Lambda_{2}$ are minimal and thus mean convex.

The unit normal vector of $\Lambda_{1}$ that points towards the interior of $N_{n, \epsilon}$ is $\vec{n}_{\Lambda_{1}}=\frac{\partial}{\partial z}$. We can extend this vector to the coordinate vector field $X=\frac{\partial}{\partial z}$ on $N_{n, \epsilon}$, and then we can compute 
the mean curvature vector of $\Lambda_{1}$ with respect to $\vec{n}_{\Lambda_{1}}$ as:

$$
\begin{aligned}
H_{\Lambda_{1}} & =-\frac{1}{2} \operatorname{div}_{N_{n, \epsilon}} X \\
& =-\left.\frac{1}{2} \frac{\partial}{\partial z}\right|_{z=-\pi} \ln \left(\operatorname{det} g_{\epsilon}\right) \\
& =-\left.\frac{1}{2} \frac{\partial}{\partial z}\right|_{z=-\pi}\left(\ln \left(\omega^{4}(z) \alpha^{2}(\phi)\right)\right) \\
& =-2 \frac{\omega^{\prime}(-\pi)}{\omega(-\pi)}=0
\end{aligned}
$$

This shows that $\Lambda_{1}$ is minimal in $N_{n, \epsilon}$, and in particular it is mean convex. A similar argument shows that $\Lambda_{2}$ is mean convex.

Finally, we will show that the vertical surfaces $\Lambda_{3}$ and $\Lambda_{4}$ are minimal in $N_{n, \epsilon}$. The inward-pointing unit normal of $\Lambda_{3}$ is $\vec{n}_{\Lambda_{3}}=\frac{1}{\omega(z) \alpha(\phi)} \frac{\partial}{\partial \theta}$, which can be extended to a vector field $X=\frac{1}{\omega(z) \alpha(\phi)} \frac{\partial}{\partial \theta}$ in all $N_{n, \epsilon}$. Then the mean curvature vector of $\Lambda_{3}$ with respect to $\vec{n}_{\Lambda_{3}}$ is:

$$
\begin{aligned}
H_{\Lambda_{3}} & =-\frac{1}{2} \operatorname{div}_{N_{n, \epsilon}} X \\
& =-\left.\frac{1}{2} \frac{\partial}{\partial \theta}\right|_{\theta=-n \pi} \ln \left(\operatorname{det} g_{\epsilon}\right)=0
\end{aligned}
$$

since the determinant of the metric does not depend on the $\theta$ coordinate. This proves that $\Lambda_{3}$ is minimal, and a similar calculation shows that $\Lambda_{4}$ is also minimal.

Therefore, the first hypothesis in Theorem 4 is also satisfied.

Consider the circles $\gamma_{3}=\{\theta=-n \pi, z=-\pi\}$ and $\gamma_{4}=\{\theta=n \pi, z=\pi\}$ : they bound an annulus in $\partial N_{n, \epsilon}$. Moreover, any homotopically nontrivial curve in that annulus is also homotopically nontrivial in $N_{n, \epsilon}$, so by Theorem 4 , there exists a smoothly embedded area-minimizing annulus $\Sigma_{n, \epsilon}^{\prime \prime}$ in $N_{n, \epsilon}$ whose boundary is $\gamma_{3} \cup \gamma_{4}$. These surfaces have the following non-intersection property:

Lemma 9 Let $R_{\theta_{0}}: N_{\epsilon} \rightarrow N_{\epsilon}$ be the isometry $\theta \rightarrow \theta+\theta_{0}$, where $\theta_{0} \neq 0$. Then

$$
R_{\theta_{0}}\left(\Sigma_{n, \epsilon}^{\prime \prime}\right) \cap \Sigma_{n, \epsilon}^{\prime \prime}=\emptyset .
$$

Proof of Lemma 9 For $\theta$ big enough (for instance, $\theta>2 n \pi$ ), it is clear that $R_{\theta}\left(\Sigma_{n, \epsilon}^{\prime \prime}\right) \cap$ $\Sigma_{n, \epsilon}^{\prime \prime}=\emptyset$. Let

$$
\theta_{0}=\sup \left\{\theta>0: R_{\theta}\left(\Sigma_{n, \epsilon}^{\prime \prime}\right) \cap \Sigma_{n, \epsilon}^{\prime \prime} \neq \emptyset\right\} .
$$

Then $R_{\theta_{0}}\left(\Sigma_{n, \epsilon}^{\prime \prime}\right) \cap \Sigma_{n, \epsilon}^{\prime \prime} \neq \varnothing$ and $R_{\theta_{0}}\left(\Sigma_{n, \epsilon}^{\prime \prime}\right)$ lies on one side of $\Sigma_{n, \epsilon}^{\prime \prime}$. By the maximum principle, Theorem 3, this implies that $R_{\theta_{0}}\left(\Sigma_{n, \epsilon}^{\prime \prime}\right)=\Sigma_{n, \epsilon}^{\prime \prime}$, which means $\theta_{0}=0$. Therefore, $R_{\theta}\left(\Sigma_{n, \epsilon}^{\prime \prime}\right) \cap \Sigma_{n, \epsilon}^{\prime \prime}=\emptyset$ for all $\theta>0$. The same argument applies to $\theta<0$.

Define $\Sigma_{n, \epsilon}^{\prime}$ to be the portion of $\Sigma_{n, \epsilon}^{\prime \prime}$ contained in $\Omega_{\epsilon}^{\prime}$. This discards the portion of our minimal surface that lies inside of the $\epsilon$-tubes, where the metric in $N_{n, \epsilon}$ is different than in $\Omega^{\prime}$, the universal cover of $\Omega \backslash\left(\gamma_{1} \cup \gamma_{2}\right)$. Then $\Sigma_{n, \epsilon}^{\prime}$ is an area-minimizing surface with boundary consisting of $\gamma_{3} \cup \gamma_{4} \cup \delta_{n, \epsilon}^{1} \cup \delta_{n, \epsilon}^{2}$, where each $\delta_{n, \epsilon}^{i}$ is some curve on the cylinder of radius $\epsilon$ centered on the axes $\gamma_{i}$. Let $\Sigma_{n, \epsilon} \subset \Omega$ be the projection of $\Sigma_{n, \epsilon}^{\prime} \subset \Omega^{\prime}$ from the universal cover to the original manifold $\Omega \subset \mathbf{S}^{2} \times \mathbf{S}^{1}$. 
By Lemma 9, $\Sigma_{n, \epsilon}^{\prime \prime}$ does not intersect any of its $\theta$-translations. Because a self-intersection of $\Sigma_{n, \epsilon}$ corresponds to the intersection of $\Sigma_{n, \epsilon}^{\prime} \subset \Sigma_{n, \epsilon}^{\prime \prime}$ with one of its $\theta$-translations, $\Sigma_{n, \epsilon}$ must be embedded in $\Omega$. More strongly, the intersection of $\Sigma_{n, \epsilon}$ with a plane $\{\phi=$ const $\}$ must be monotone in the vertical coordinate $z$.

At this point in our construction, the surfaces $\Sigma_{n, \epsilon}$ are essentially embedded minimal surfaces in $\Omega$ that wrap $n$ times around $\epsilon$-tubes centered on the axes $\gamma_{1}$ and $\gamma_{2}$ and whose boundary is the union of fixed geodesics $\gamma_{3}, \gamma_{4}$, and some curves contained in these $\epsilon$-tubes. The next step is to let $\epsilon \rightarrow 0$ to create a sequence of minimal surfaces $\tilde{\Sigma}_{n}$ that wrap $n$ times around the axes $\gamma_{1}$ and $\gamma_{2}$ and whose boundaries are $\cup_{i=1}^{4} \gamma_{i}$. These surfaces are essentially "half-helicoids" which we will extend via Schwarz reflection (Theorem 6) into our final sequence $\Sigma_{n}$.

Specifically, to construct each $\tilde{\Sigma}_{n}$, we need area and curvature bounds on $\Sigma_{n, \epsilon}$ independent of $\epsilon$. First we obtain a curvature bound: let $\Omega_{\epsilon_{0}}$ be the complement of the $\epsilon_{0}$-neighborhood of $\cup_{i=1}^{4} \gamma_{i}$. Clearly, $\cup_{\epsilon_{0}>0} \Omega_{\epsilon_{0}}=\Omega \backslash\left(\cup_{i=1}^{4} \gamma_{i}\right)$. Fix $\epsilon_{0}>0$. Because each $\Sigma_{n, \epsilon}$ is isometric to a subset of the stable minimal surfaces $\Sigma_{n, \epsilon}^{\prime \prime}$, we have the interior curvature bound

$$
\sup _{\Omega_{\epsilon_{0}}}\left|A\left(\Sigma_{n, \epsilon}\right)\right|^{2} \leq C\left(\epsilon_{0}\right)
$$

for any $\epsilon<\epsilon_{0} / 2$ and some constant $C\left(\epsilon_{0}\right)$ dependent only on $\epsilon_{0}$.

Similarly, we can obtain a uniform area bound for $\Sigma_{n, \epsilon} \cap \Omega_{\epsilon_{0}}$. Each $\Sigma_{n, \epsilon}$ is the projection of an area-minimizing surface $\Sigma_{n, \epsilon}^{\prime} \subset \Omega^{\prime}$. As before, let $\Omega_{\epsilon_{0}}^{\prime}$ be the universal cover of $\Omega_{\epsilon_{0}}$ (or equivalently, $\Omega_{\epsilon_{0}}^{\prime}$ is the complement in $\Omega^{\prime}$ of the $\epsilon_{0}$-neighborhoof of $\left.\partial \Omega^{\prime}\right)$. If we construct a surface consisting of a fixed surface $\Sigma_{n, \epsilon_{0}}^{\prime} \cap \Omega_{\epsilon_{0}}^{\prime}$ plus the piece of $\partial \Omega_{\epsilon_{0}}^{\prime}$ between $\partial \Sigma_{n, \epsilon}^{\prime} \cap \Omega_{\epsilon_{0}}^{\prime}$ and $\partial \Sigma_{n, \epsilon_{0}}^{\prime} \cap \Omega_{\epsilon_{0}}^{\prime}$, then this new surface has bigger area than $\Sigma_{n, \epsilon}^{\prime} \cap \Omega_{\epsilon_{0}}^{\prime}$. We can then bound uniformly the area of $\Sigma_{n, \epsilon}^{\prime} \cap \Omega_{\epsilon_{0}}^{\prime}$ by the area of $\Sigma_{n, \epsilon_{0}}^{\prime} \cap \Omega_{\epsilon_{0}}^{\prime}$ plus the area of $\partial \Omega_{\epsilon_{0}}^{\prime}$. Now since the area of the projection $\Sigma_{n, \epsilon}$ is the same as the area of $\Sigma_{n, \epsilon}^{\prime}$ (because $\Omega$ and $\Omega^{\prime}$ are isometric and $\Sigma_{n, \epsilon}$ is embedded, that is, there is no self-intersections), this gives us a bound of the area of $\Sigma_{n, \epsilon}$ uniform in $\epsilon$.

This area bound combined with the previous interior curvature bound implies that for any fixed $\epsilon_{0}>0$, we can find a sequence of $\Sigma_{n, \epsilon}$ that converges when $\epsilon \rightarrow 0$ to a locally areaminimizing embedded surface in $\Omega_{\epsilon_{0}}$. By letting $\epsilon_{0} \rightarrow 0$ and taking a diagonal sequence, we can extract a subsequence $\left\{\Sigma_{n, \epsilon_{j}}\right\}$ that converges to an embedded minimal surface $\tilde{\Sigma}_{n}$ in $\Omega \backslash\left(\cup_{i=1}^{4} \gamma_{i}\right)$. Additionally, $\partial \Sigma_{n, \epsilon_{j}}$ converges pointwise to $\cup_{i=1}^{4} \gamma_{i}$.

So far, we have only shown that $\tilde{\Sigma}_{n}$ is embedded away from $\gamma_{1}$ and $\gamma_{2}$. However, consider the portion of $\partial \Sigma_{n, \epsilon_{j}}$ that lies on the cylinders $\left\{\phi=\epsilon_{j}, \pi-\epsilon_{j}\right\}$. By Lemma 9, the projection of these portions onto $\gamma_{1}$ and $\gamma_{2}$ must be monotone (otherwise, $\Sigma_{n, \epsilon_{j}}^{\prime}$ would intersect one of its $\theta$-translations; see Fig. 3). In particular, this implies that $\tilde{\Sigma}_{n}$ is embedded up to the boundary $\cup_{i=1}^{4} \gamma_{i}$.

To prove that this embedding is non-singular at a point $x \in \gamma_{1} \cup \gamma_{2}$, choose a small intrinsic neighborhood $U \subset \tilde{\Sigma}_{n}$ of $x$. As $\tilde{\Sigma}_{n}$ is the limit of the area-minimizing surfaces $\Sigma_{n, \epsilon}, U$ will be area-minimizing provided that it is sufficiently small. We can also choose $U$ to have smooth boundary. Therefore, we can apply Theorem 5 to show that $U$ is $C^{1, \alpha}$ up to $\partial U \cap\left\{\gamma_{1} \cup \gamma_{2}\right\}$.

Thus, the surfaces $\tilde{\Sigma}_{n}$ have boundary on $\{z= \pm \pi\} \cup \gamma_{1} \cup \gamma_{2}$. The final step is to use Theorem 6 to reflect them across the axes $\gamma_{1}$ and $\gamma_{2}$. In this case the isometry $G$ is the rotation $R_{\pi}: \theta \mapsto \theta+\pi$ around the polar axes. The new surfaces $\Sigma_{n}$ are cylinders in $\Omega$ with boundary in $\partial \Omega=\{z=-\pi\} \cup\{z=\pi\}$. Observe that $\Sigma_{n} \backslash\left(\gamma_{1} \cup \gamma_{2}\right)=\tilde{\Sigma}_{n} \cup G\left(\tilde{\Sigma}_{n}\right)$, and when we lift $G\left(\tilde{\Sigma}_{n}\right)$ to $\Omega^{\prime}$ we obtain just a $\theta$-translation of $\Sigma_{n}^{\prime}$. By Lemma 9, we can show 

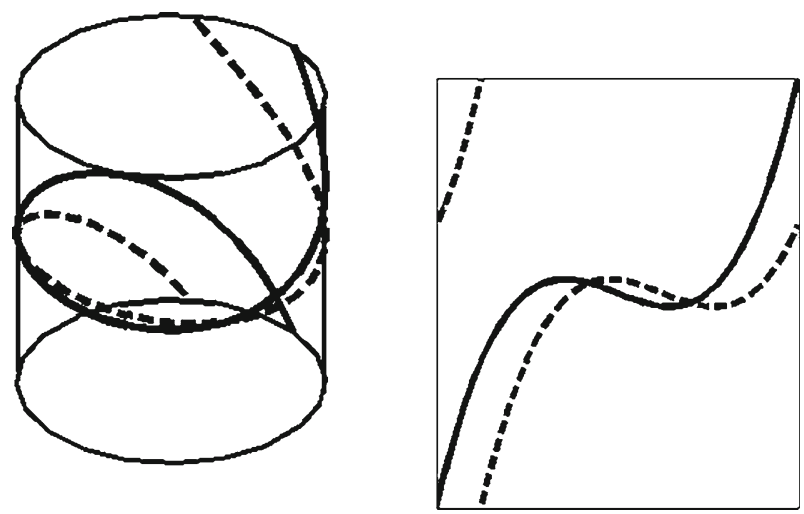

Fig. 3 If the boundary curve $\Sigma_{n, \epsilon_{j}} \cap\left\{\phi=\epsilon_{j}, \pi-\epsilon_{j}\right\}$ is not monotone, then it intersects its $\theta$-translation, that is, its rotation around the polar axes

that $\tilde{\Sigma}_{n} \cap G\left(\tilde{\Sigma}_{n}\right) \subset \gamma_{1} \cup \gamma_{2}$, and therefore each $\Sigma_{n}$ is a smooth embedded minimal surface in $\Omega$.

\section{The limit of the sequence $\Sigma_{n}$}

We will now finish the proof of Theorem 1 by showing that the sequence $\Sigma_{n}$ converges smoothly to a minimal lamination away from the axes $\gamma_{1}$ and $\gamma_{2}$ and that the limit lamination has two helicoid-like singularities on the strictly stable 2 -sphere $\Gamma$.

We say that a sequence of surfaces $\Sigma_{n} \subset M$ is uniformly locally simply connected(ULSC) if for each $x \in M$, there exists a constant $r_{0}>0$ (depending on $x$ ), so that for all $r \leq r_{0}$ and every surface $\Sigma_{n}$ each connected component of $B_{r}(x) \cap \Sigma_{n}$ is a disk.

The existence of the limit lamination is given by the following lemma (see [16], Lemma II.1.2 in [17] and Theorem 4.2 in [9]):

Lemma 10 Let $M$ be a compact three-dimensional manifold with boundary and let $\Sigma_{n}$ be a sequence of compact embedded minimal surfaces in $M$ with $\partial \Sigma_{n} \subset \partial M$. Let

$$
S=\left\{x \in M: \sup _{B_{r}(x) \cap \Sigma_{j}}|A|^{2} \rightarrow \infty \text { for all } r>0\right\}
$$

and suppose the sequence is ULSC in a neighborhood of S. Then after passing to a subsequence, $\stackrel{\circ}{\Sigma}_{n} \backslash S$ will converge on compact subsets to a lamination $\mathcal{L} \subset \stackrel{M}{S}$ s with minimal leaves.

Proof For each compact subset $K \in M \backslash S$, there is an open covering of $K$ by finitely many balls where the curvatures of the $\Sigma_{j}$ 's are bounded uniformly in $j$ in the concentric double balls (by the definition of $S$ ). The claim then follows from Proposition 7 and a diagonal argument.

To apply Lemma 10 to our case, we need to show that $\left\{\Sigma_{n}\right\}$ is ULSC in a neighborhood of the singular set $S$. Since the "half-helicoids" $\tilde{\Sigma}_{n}$ are stable, we have uniform curvature bounds on $\Sigma_{n}$ depending only on the distance to the boundary and the axes $\gamma_{1}$ and $\gamma_{2}$. By 
slightly shrinking $\Omega$, we can obtain uniform curvature bounds away from the polar axes. Therefore the singular set $S$ must be contained in $\gamma_{1} \cup \gamma_{2}$.

A point $y$ in the singular set $S$ is not ULSC if and only if the injectivity radius on the sequence go to 0 at that point, that is, there is a sequence of points $y_{n} \in \Sigma_{n}$ with $y_{n} \rightarrow y$ and $\operatorname{inj}\left(y_{n}\right) \rightarrow 0$ (see Part IV in [17], Proposition I.0.19 in [15]). If $d_{\Sigma_{n}}\left(y_{n}, y\right)$ is bounded away from zero (ie, the sequence $y_{n}$ converges to $y$ extrinsically but not intrinsically), then standard curvature bounds for stable minimal surfaces (see, for example, Theorem 2.7 of [7]) imply that the injectivity radius of $y_{n}$ is bounded away from zero. Therefore, we only need to prove a lower bound on injectivity radius for all points in an intrinsic neighborhood of the axes.

Consider an intrinsic $r$-neighborhood of the axis $D_{r}^{n}\left(\gamma_{1}\right)=\left\{x \in \Sigma_{n}: d_{\Sigma_{n}}\left(x, \gamma_{1}\right)<r\right\}$. We can write $D_{r}^{n}\left(\gamma_{1}\right)=\tilde{D}_{r}^{n}\left(\gamma_{1}\right) \cup G\left(\tilde{D}_{r}^{n}\left(\gamma_{1}\right)\right)$, where $\tilde{D}_{r}^{n}\left(\gamma_{1}\right)$ is the corresponding $r$-neighborhood of $\gamma_{1}$ in $\tilde{\Sigma}_{n}$, and $\tilde{D}_{r}^{n}\left(\gamma_{1}\right) \cap G\left(\tilde{D}_{r}^{n}\left(\gamma_{1}\right)\right)=\gamma_{1}$. Since both $\tilde{\Sigma}_{n}$ and $G\left(\tilde{\Sigma}_{n}\right)$ are disks, $\tilde{D}_{r}^{n}\left(\gamma_{1}\right) \backslash \gamma_{1}$ and $G\left(\tilde{D}_{r}^{n}\left(\gamma_{1}\right)\right) \backslash \gamma_{1}$ are disjoint disks, and therefore $D_{r}^{n}\left(\gamma_{1}\right)$ is topologically a disk. Thus, in smaller neighborhood $D_{\frac{r}{2}}\left(\gamma_{1}\right)$, the injectivity radius is bounded below by $\frac{r}{2}$, and this implies that the sequence is ULSC in the axis $\gamma_{1}$. The same argument applies to $\gamma_{2}$.

Once we have the minimal lamination $\mathcal{L}$, there are only two possibilities for each leaf; either the leaf is a closed surface or it spirals infinitely into a closed surface. The only closed surface in $\Omega$ is the strictly stable 2 -sphere $\Gamma$, and so our lamination will consist of $\Gamma$ and two minimal surfaces that spiral into it.

Theorem 11 After passing to a subsequence, the sequence $\left\{\Sigma_{n}\right\}$ converges to a lamination $\mathcal{L}$ of minimal surfaces consisting of the strictly stable 2 -sphere $\Gamma$ and two embedded minimal surfaces that lie on opposite sides of and spiral into $\Gamma$.

Proof By Lemma 10, a subsequence of $\left\{\Sigma_{n}\right\}$ will converge to a minimal lamination $\mathcal{L}$ away

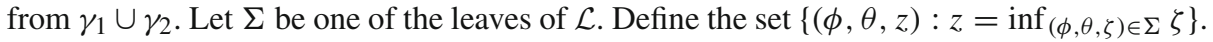
Because $\Sigma$ is embedded and $\mathcal{L}$ is a (closed) lamination, this set defines a leaf of $\mathcal{L}$ which is a closed minimal graph over $\Gamma$ in $M$. By Lemma 2, there are no non-trivial minimal graphs over $\Gamma$ in $\Omega$, which implies that $\Sigma=\Gamma$ or that $\Sigma$ must infinitely spiral into $\Gamma$. In either case, this shows that $\Gamma \in \mathcal{L}$.

Because each $\Sigma_{n}$ has boundary in both boundary components of $\Omega$, the lamination $\mathcal{L}$ must contain at least one leaf that does not equal to $\Gamma$ and therefore must spiral into $\Gamma$. Since each $\Sigma_{n}$ is connected and crosses $\Gamma$ but no leaf of $\mathcal{L}$ can cross $\Gamma$, there must be exactly two such spiraling surfaces, one on each side of $\Gamma$.

Theorem 1 now follows immediately.

Proof of Theorem 1 We can calculate the scalar curvature of a warped product on $M$ as

$$
\operatorname{Scal}_{M}=-2 \frac{\omega^{\prime \prime}}{\omega}+\frac{1-\left(\omega^{\prime}\right)^{2}}{\omega^{2}} .
$$

In particular, we can choose $\omega \in C^{\infty}\left(\mathbf{S}^{1}\right)$ satisfying the four properties listed before and with positive scalar curvature. An example of such a function would be

$$
\omega(z)=-\frac{1}{4} \cos z+\frac{5}{4} .
$$


Acknowledgements We would like to thank our adviser Tobias Colding for his many helpful suggestions. We are also thankful to the referee for the many comments that have greatly improved the exposition of this paper.

Open Access This article is distributed under the terms of the Creative Commons Attribution Noncommercial License which permits any noncommercial use, distribution, and reproduction in any medium, provided the original author(s) and source are credited.

\section{References}

1. Alarcon, A., Ferrer, L., Martin, F.: Density theorems for complete minimal surfaces in $\mathbf{R}^{3}$. http://arxiv. org/math.DG/0603737

2. Baldi, A.: Weighted BV functions. Houston J. Math. 27(3), 683-705 (2001)

3. Choi, H., Schoen, R.: The space of minimal embeddings of a surface into a three-dimensional manifold of positive Ricci curvature. Invent. Math. 81(3), 387-394 (1985)

4. Choi, H., Wang, A.: A first eigenvalue estimate for minimal hypersurfaces. J. Differ. Geom. 18(3), 559562 (1983)

5. Colding, T.H., De Lellis, C.: Singular limit laminations, Morse index, and positive scalar curvature. Topology 44(1), 24-45 (2005)

6. Colding, T.H., De Lellis, C., Minicozzi, W.P.: Three circles theorems for Schrödinger operators on cylindrical ends and geometric applications (preprint)

7. Colding, T.H., Minicozzi, W.P.: Minimal Surfaces, Courant Lecture Notes in Mathematics, vol. 4. New York University, Courant Institute of Mathematical Sciences, New York (1999)

8. Colding, T.H., Minicozzi, W.P.: Embedded minimal disks: proper versus nonproper-global versus local. Trans. Am. Math. Soc. 356(1), 283-289 (1998)

9. Colding, T.H., Minicozzi, W.P.: Embedded minimal surfaces without area bounds in 3-manifolds. Geometry and Topology: Aarhus (1998), Contemp. Math. 258, Am. Math. Soc., Providence (2000)

10. Colding, T.H., Minicozzi, W.P.: Finiteness of embedded minimal surfaces in 3-manifolds with positive scalar curvature (in preparation)

11. Colding, T.H., Minicozzi, W.P.: Multivalued minimal graphs and properness of disks. Int. Math. Res. Not. 21, 1111-1127 (2002)

12. Colding, T.H., Minicozzi, W.P.: The Calabi-Yau conjectures for embedded surfaces. Ann. Math. (2) (to appear)

13. Colding, T.H., Minicozzi, W.P.: The space of embedded minimal surfaces of fixed genus in a 3-manifold. I. Estimates off the axis for disks. Ann. Math. (2) 160(1), 27-68 (2004)

14. Colding, T.H., Minicozzi, W.P.: The space of embedded minimal surfaces of fixed genus in a 3-manifold. II. Multi-valued graphs in disks. Ann. Math. (2) 160(1), 69-92 (2004)

15. Colding, T.H., Minicozzi, W.P.: The space of embedded minimal surfaces of fixed genus in a 3-manifold. III. Planar Domains Ann. Math. (2) 160(2), 523-572 (2004)

16. Colding, T.H., Minicozzi, W.P.: The space of embedded minimal surfaces of fixed genus in a 3-manifold. IV. Locally simply connected. Ann. Math. (2) 160(2), 573-615 (2004)

17. Colding, T.H., Minicozzi, W.P.: The space of embedded minimal surface of fixed genus in a 3-manifold. V. Fixed genus, preprint (2005). http://www.arxiv.org/pdf/math/0509647

18. Collin, P., Rosenberg, H.: Notes sur la démonstration de N Nadirashvili des conjectures de Hadamard et Calabi-Yau. Bull. Sci. Math. 123(7), 563-575 (1999)

19. Federer, H.: The singular sets of area minimizing rectifiable current with codimension one and of area minimizing flat chains modulo two with arbitrary codimension. Bull. A.M.S. 76, 767-771 (1980)

20. Gilbarg, D., Trudinger, N.: Elliptic Partial Differential Equations of Second Order, 2nd edn. Springer, New York (1983)

21. Giusti, E.: Minimal surfaces and functions of bounded variation. Monographs in Mathematics, vol. 80. Birkhäuser, Basel (1984)

22. Han, Q., Lin, F.: Elliptic Partial Differential Equations, Courant Lecture Notes in Mathematics, vol. 1. New York University, Courant Institute of Mathematical Sciences, New York (1997)

23. Hardt, R., Simon, L.: Boundary regularity and embedded solutions for the oriented Plateau problem. Ann. Math. (2) 110(3), 439-486 (1979)

24. Hass, J., Norbury, P., Rubinstein, J.: Minimal spheres of arbitrarily high Morse index. Comm. Anal. Geom. 11(3), 425-439 (2003)

25. Hass, J., Scott, P.: The existence of least area surfaces in 3-manifolds. Trans. Am. Math. Soc. 310(1), 87-114 (1988) 
26. Hoffman, D., Weber, M., Wolf, M.: An embedded genus one helicoid. Ann. Math. (2) (to appear)

27. Hoffman, D., Weber, M., Wolf, M.: An embedded genus-one helicoid. PNAS 102, 16566-16568 (2005)

28. Hoffman, D., White, B.: Genus-one helicoids from a variational point of view. Comment. Math. Helvetici (to appear)

29. Hsiang, W., Lawson, H.: Minimal submanifolds of low cohomogeneity. J. Differ. Geom. 5, 1-38 (1971)

30. Jorge, L., Xavier, F.: A complete minimal surface in $\mathbf{R}^{3}$ between two parallel planes. Ann. Math. (2) 112(1), 203-206 (1980)

31. Martin, F., Morales, S.: Complete proper minimal surfaces in convex bodies of $\mathbf{R}^{3}$. Duke Math. J. 128(3), 559-593 (2005)

32. Meeks, W.: Regularity of the singular set in the Colding-Minicozzi lamination theorem. Duke Math. J. 123(2), 329-334 (2004)

33. Meeks, W.: The limit lamination metric for the Colding-Minicozzi minimal lamination. Illinois J. Math. 49(2), 645-658 (2005)

34. Meeks, W., Perez, J.: Conformal properties in classical minimal surface theory. Surveys in Differential Geometry, vol. IX, pp. 275-335, Surv. Differ. Geom., IX, Int. Press, Somerville, MA (2004)

35. Meeks, W., Rosenberg, H.: The uniqueness of the helicoid. Ann. Math. (2) 161(2), 727-758 (2005)

36. Meeks, W., Weber, M.: Bending the helicoid, preprint (2005). http://arxiv.org/abs/math.DG/0511387

37. Meeks, W., Yau, S.T.: The existence of embedded minimal surfaces and the problem of uniqueness. Math. Z. 179(2), 151-168 (1982)

38. Morgan, F.: Regularity of isoperimetric hypersurfaces in Riemannian manifolds. Trans. Am. Math. Soc. 355(12), 5041-5052 (2003)

39. Nadirashvili, N.: Hadamard's and Calabi-Yau's conjectures on negatively curved and minimal surfaces. Invent. Math. 126(3), 457-465 (1996)

40. Osserman, R.: A Survey of Minimal Surfaces. Dover Publications Inc., New York (1986)

41. Perez, J.: Limits by rescalings of minimal surfaces: minimal laminations, curvature decay and local pictures. Notes for the workshop "Moduli Spaces of Properly Embedded Minimal Surfaces", American Institute of Mathematics, Palo Alto, CA (2005)

42. Solomon, B., White, B.: A strong maximum principle for varifolds that are stationary with respect to even parametric elliptic functionals. Indiana J. Math. 38, 683-691 (1989)

43. White, B.: The space of minimal submanifolds for varying Riemannian metrics. Indiana Univ. Math. J. 40(1), 161-200 (1991)

44. White, B.: New applications of mapping degrees to minimal surface theory. J. Differ. Geom. 29(1), 143-162 (1989)

45. Yang, P., Yau, S.T.: Eigenvalues of the Laplacian of compact Riemann surfaces and minimal submanifolds. Ann. Scuola Norm. Sup. Pisa Cl. Sci. 7(1), 55-63 (1980) 